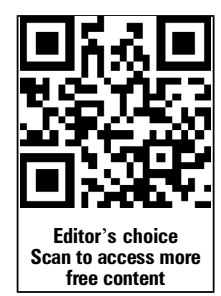

${ }^{1}$ Department of Dermatology, Great Ormond Street Hospital for Children, London, UK ${ }^{2}$ Department of Plastic and Reconstructive Surgery, Great Ormond Street Hospital for Children, London, UK

\section{Correspondence to}

Dr Lea Solman, Department of Paediatric Dermatology, Great Ormond Street Hospital, Level 6 , Southwood Building, London WC1N 3JH, UK lea.solmankosuti@@gosh.nhs. uk

LS and AM contributed equally.

Received 29 April 2014 Revised 19 July 2014 Accepted 21 July 2014 Published Online First 14 August 2014

\title{
Propranolol for infantile haemangiomas: single centre experience of 250 cases and proposed therapeutic protocol
}

\author{
Lea Solman, ${ }^{1}$ Amera Murabit, ${ }^{2}$ Maria Gnarra, ${ }^{1}$ John I Harper, ${ }^{1}$ Samira B Syed, ${ }^{1}$ \\ Mary Glover ${ }^{1}$
}

\section{ABSTRACT}

Objective To assess the safety and efficacy of systemic propranolol for the treatment of complicated infantile haemangiomas.

Design Retrospective review of case notes of paediatric patients treated with propranolol for complicated infantile haemangiomas.

Setting Tertiary care children's hospital.

Patients All paediatric patients with complicated infantile haemangiomas who commenced treatment with propranolol from July 2008 to December 2011 and have completed treatment for at least 3 months.

Results 250 patients were treated with propranolol; $34.4 \%$ were premature and $5.6 \%$ postmature. Indications for propranolol included: vision compromise $(42.0 \%)$, bleeding and/or ulceration (30.4\%) airway obstruction $(8.8 \%)$, feeding difficulty $(8.4 \%)$, risk of permanent disfigurement $(4.4 \%)$ and other $(6 \%)$ (nasal obstruction, auditory canal obstruction, large haemangioma, compression of neck structure and spinal cord). Median age at beginning of treatment was 4.5 months. Median age at end of treatment was 16.7 months. Median length of therapy was 11.8 months. Adverse effects (such as wheezing, worsening of ulceration, sleep disturbance, diarrhoea) occurred in 38 patients $(15.2 \%)$, leading to modifications in management in 26 patients (10.4\%). 240 patients (96\%) had good to excellent response to treatment. 20 patients (8\%) experienced regrowth of the haemangioma on cessation of propranolol and six patients $(2.4 \%)$ required propranolol to be restarted.

Conclusions In appropriately selected patients, propranolol is a safe and effective treatment for infantile haemangiomas.

\section{INTRODUCTION}

Infantile haemangiomas (IHs) are the most common benign vascular tumours of infancy affecting up to $10 \%$ of children, with a female to male ratio of $3: 1^{1}$ and a higher prevalence in the Caucasian population. ${ }^{2}$ Prematurity ${ }^{3}$ and low birth weight $(<1500$ grams) are considered potential predisposing factors. IHs are characterised by a triphasic clinical and histological evolution with rapid growth (proliferative phase) followed by a plateau period and a slow spontaneous involution (involutive phase). ${ }^{4}$

More than $60 \%$ of IHs occur on the face, head and neck. ${ }^{5}$ Although benign, the involvement of the eyelids, nasal tip, lips and ears may endanger breathing, feeding and vision or lead to irreversible disfigurement. ${ }^{6}$ Bleeding, ulceration and subsequent

\section{What is already known on this topic?}

- Infantile haemangiomas are the most common paediatric vascular tumours and most do not require intervention.

- Propranolol has become a first-line treatment in the management of infantile haemangiomas.

\section{What this study adds?}

Propranolol is a safe and effective treatment of infantile haemangiomas. The adverse effects are mild if the patients are carefully selected, monitored and parents adequately educated about the treatment.

- We have simplified our treatment protocol, reducing the need for pretreatment blood testing, ECG, echocardiogram, and ongoing monitoring of blood pressure and heart rate.

infection can occur in up to $20 \%$ of cases. $^{7}$ Early recognition and treatment of critical lesions help in preventing or minimising complications.

Before 2008, treatment for IH had included systemic and intralesional corticosteroids ${ }^{8}$ and $\alpha$-interferon, ${ }^{9}$ which were associated with significant adverse effects. In 2008, the first report of the successful use of propranolol radically changed therapy and, since then, propranolol has become the first-line therapeutic agent in the management of IHs. ${ }^{10}$

Propranolol is a non-selective $\beta$-adrenergic receptor blocker traditionally used for cardiovascular and anxiety disorders. Although the specific mechanism of action on IH remains largely unknown, propranolol appears to produce clinical improvement by inducing vasoconstriction and apoptosis and decreasing expression of pro-angiogenic factors. ${ }^{11}$

While propranolol's mechanism of action on $\mathrm{IH}$ is still under investigation, it has been shown to induce a better and faster response in IH management than systemic steroids, and is associated with fewer adverse effects. ${ }^{12} 13$

The purpose of this paper is to present our experience with propranolol for the management of IH in a specialised tertiary care hospital. For this 
group of patients, a therapeutic protocol was designed when there was very limited experience of propranolol for this indication. ${ }^{14}$ As a result of our experience, we have modified this protocol, reducing the need for pretreatment blood testing, ECG, echocardiogram (ECHO), and ongoing monitoring of blood pressure (BP) and heart rate (HR).

\section{PATIENTS AND METHODS}

A retrospective case notes review was performed on 250 paediatric patients treated with propranolol for $\mathrm{IH}$ at our hospital from July 2008 to December 2011. We identified all patients diagnosed with $\mathrm{IH}$ for which propranolol was indicated and prescribed according to our protocol, but only those who had completed their course of treatment at least 3 months before were included.

Pretreatment investigations included a full blood count (FBC), urea, creatine, electrolytes, urinalysis, liver function tests (LFT), glucose, ECG, ECHO and clinical photography. Administration of oral liquid propranolol was dosed based on patient weight starting from an initial dose of $1 \mathrm{mg} / \mathrm{kg} /$ day, divided in three equal doses, for the first week followed by $2 \mathrm{mg} / \mathrm{kg} / \mathrm{day}$, divided in three equal doses, in the second week and then continuous dose adjustments according to the child's weight at each clinical review. The initiation dose for children born prematurely or diagnosed with other comorbidities was $0.5 \mathrm{mg} / \mathrm{kg} /$ day divided in three equal doses for the first week, followed by $1 \mathrm{mg} / \mathrm{kg} /$ day divided in three equal doses in the second week. Further increases were based on clinical judgement. Children were observed inhospital for 2-4 h with initiation and initial dosage elevation of propranolol therapy (premature/comorbid patients were observed for at least 4-8 h). Observations included assessment of general well-being, HR and BP monitoring every $30 \mathrm{~min}$. These observations were compared with the age-related referenced data. As outpatients, BP and HR were checked on a weekly basis for the first 4-6 weeks, then fortnightly for the remainder of the therapeutic period. For the measurements taken out of our hospital, we recommended maintaining a BP greater than $70 \mathrm{~mm} \mathrm{Hg}$ systolic and $35 \mathrm{~mm} \mathrm{Hg}$ diastolic, with an HR above 100/min and notifying our departmental nurses and/or their local doctor if there are any concerns. Parents/caregivers were made aware of known side effects prior to commencement of therapy, including bradycardia, heart failure, hypotension, cardiac conduction disorder, bronchospasm, peripheral vasoconstriction, weakness and fatigue, sleep disturbance and hypoglycaemia. Parents were advised to stop the propranolol in case of serious adverse effect and to contact a member of our team.

Assessment included evaluation by the clinician as to whether there was no response, poor, good or excellent response, plus photographic documentation of $\mathrm{IH}$ at each visit. We defined excellent response as 75\%-100\% improvement, good response as $50 \%-75 \%$ improvement, poor response as $25 \%-50 \%$ improvement and no response as less than 25\% improvement. Propranolol therapy was terminated, according to individual clinical response, by halving the dose for 2 weeks and further halving it for another 2 weeks and then stopped, so that propranolol therapy was gradually reduced and stopped over the course of about 1 month.

Data regarding patient demographics, haemangioma characteristics, indications for propranolol treatment, associated investigations, management modifications, concomitant therapies, side effects and overall effectiveness were compiled into a separate database and analysed.

\section{RESULTS}

In all, 201 patients were girls and 49 were boys. About a third of patients (86 patients) were born prematurely, and 14 patients were born after $\geq 42$-week gestation.

IHs were located on the face (181 lesions), head and neck (57 lesions), trunk (36 lesions) and extremities (23 lesions). Concomitant visceral lesions were documented in nine patients (3.6\%), seven of which were localised to the liver and the remaining two were intraspinal.

Primary indications for propranolol included: vision compromise (42.0\%), bleeding and/or ulceration (30.4\%), airway obstruction $(8.8 \%)$, feeding difficulty $(8.4 \%)$, risk of permanent disfigurement (4.4\%), nasal obstruction (four patients, 1.6\%), auditory canal obstruction (three patients, 1.2\%), widespread haemangiomas (four patients, $1.6 \%$ ), compression of neck structures (three patients, $1.2 \%$ ) and compression of spinal cord (one patient, 0.4\%).

Pretreatment blood test investigations and urinalysis did not reveal abnormalities contraindicating propranolol administration in any of our patients. Pretreatment ECG testing showed no signs of cardiac rhythm irregularities (not including sinus tachycardia).

Pretreatment ECHO was normal in 208 patients (83.2\%), clinically inconsequential in 34 patients $(13.6 \%)$ and abnormal in eight patients (3.2\%). Pathologies included VSD (four patients), large PDA (two patients), aortic coarctation (one patient) and tetralogy of Fallot (one patient). Four patients required a surgical procedure (VSD closure in one patient, aortic coarctation repair in one patient, PDA ligation in one patient and tetralogy of Fallot repair in one patient).

The cardiac defects were already known in seven of these patients. One patient presented to the department of paediatric dermatology first with a large segmental facial haemangioma and pretreatment ECHO revealed aortic coarctation.

The inhospital monitoring period was uneventful in all of the patients presented in this series.

The median age at the beginning of propranolol treatment was 4.5 months (range 0.7-48.1 months). The median age at the end of propranolol treatment was 16.7 months (range 5.561.1 months). The median length of propranolol therapy was 11.8 months (range $0.2-43.0$ months). In all, 65 patients started their treatment after the age of 6 months.

Overall, 29 patients (11.6\%) received oral prednisolone. All had presented with functional lesions (obstruction of airway or vision) and prescribed prednisolone either before or in the early stages of usage of propranolol for IH.

Table 1 Adverse effects $(n=250)$

\begin{tabular}{ll}
\hline Observed adverse effect & N (\%) \\
\hline $\begin{array}{l}\text { Wheezing } \\
\text { Usually bronchial hyperactivity in the setting of viral respiratory } \\
\text { infection }\end{array}$ & $17(6.8)$ \\
Sleep disturbance & \\
Worsening of the ulceration & $9(3.6)$ \\
Asymptomatic bradycardia & $4(1.6)$ \\
Peripheral cyanosis & $2(0.8)$ \\
Vomiting & $2(0.8)$ \\
Diarrhoea & $2(0.8)$ \\
Asymptomatic hypotension & $1(0.4)$ \\
\hline & $1(0.4)$ \\
\hline
\end{tabular}




\section{Original article}

Adverse events were observed in 38 patients (15.2\%) (table 1). Two patients had asymptomatic bradycardia and one patient had asymptomatic hypotension.

A total of 26 patients (10.4\%) had alterations in therapy including dosage reduction/alterations due to side effects (eight patients, $3.2 \%$ ), temporary withholding of propranolol (six patients, $2.4 \%$ ) and premature cessation of treatment (12 patients, $4.8 \%$ ).

Overall, 230 patients (92.0\%) did not experience enlargement or recurrence of the lesion after propranolol therapy was stopped. Of the 20 patients $(8.0 \%)$ who did, $6(2.4 \%)$ required propranolol to be restarted and the other 14 (5.6\%) showed reduction in size over time, without restarting propranolol. The average length of treatment in the patients with rebound growth was 46 days shorter than in patients with no rebound growth.
Overall, outcome after stopping the propranolol was graded as no response, poor, good or excellent. A total of 240 patients (96.0\%) had good or excellent overall outcome.

\section{DISCUSSION}

Our experience has shown propranolol to be an effective treatment for IH. Although systemic $\beta$-blockers have the potential to cause adverse effects, the complication rate in our study was low and the adverse effects were not life threatening. In all, $6.8 \%$ of our patients experienced wheezing, usually occurring with viral respiratory infection. Wheezing is otherwise common in infancy, with a reported prevalence of $28.5 \%-32.0 \% .^{15} 16$ Adverse events reported in recent literature, including hypoglycaemia and hyperkalaemia, ${ }^{17-19}$ were not experienced in our

Table 2 Treatment protocol

\section{Before starting propranolol}

- Full clinical history and examination

including $\mathrm{HR}, \mathrm{BP}$ and $\mathrm{SpO}_{2}$

- Parents' education and written information given

- Clinical photography

- ECHO and ECG in selected patients (please see table below)

ECG ECG and ECHO

If the HR is below normal for age History of arrhythmia or arrhythmia on examination

Family history of congenital heart condition, arrhythmia or maternal history of connective tissue disease

\section{Dosage regime}

Week 1

Week 2

Patients with suspected PHACE syndrome

$1 \mathrm{mg} / \mathrm{kg} /$ day divided into three equal doses

$2 \mathrm{mg} / \mathrm{kg} /$ day divided into three equal doses

$0.5 \mathrm{mg} / \mathrm{kg} /$ day and very cautious increase of the dose in small increments until MRA excludes arterial anomalies of head and neck

Observation and monitoring

Weight $>3.5 \mathrm{~kg}$ and no comorbidities

Weight $<3.5 \mathrm{~kg}$ and/or comorbidities

$\mathrm{BP}$ and $\mathrm{HR}$ immediately before the dose and every $30 \mathrm{~min}$ for $2 \mathrm{~h}$

$\mathrm{BP}$ and HR immediately before the dose and every $30 \mathrm{~min}$ for $4 \mathrm{~h}$ or longer

\section{Other investigations*}

\section{Investigations}

\section{Group of patients}

Blood tests:

Thyroid function test

Liver function tests

Full blood count

Abdominal ultrasound

MRA brain and neck

MRI spine

Liver haemangioma

Parotid haemangioma

PHACE syndrome

Liver haemangioma

Bleeding haemangiomas

More than 10 cutaneous haemangiomas

Perianal and perineal haemangioma crossing the midline and/or extending into gluteal cleft

Large segmental haemangioma of head and neck with suspected PHACE syndrome

Plaque haemangioma in lumbosacral area crossing the midline or perianal/perineal haemangioma extending into gluteal cleft

\section{Referrals}

\section{ENT team}

Ophthalmology team

Suspected airway haemangioma

Peri-ocular haemangioma

\section{Continuation of the treatment}

\section{Reviews}

Increments

Treatment stopping
Increments greater than $0.5 \mathrm{mg} / \mathrm{kg} /$ day should include monitoring of $\mathrm{HR}$ and BP for $2 \mathrm{~h}$

Usually at 12-14 months of age but can be longer

Gradual dose reduction over 2-4 weeks
4-6 weeks after starting treatment, then 3-4 monthly

\section{*Propranolol can be commenced while the investigations/results are pending.}

$\mathrm{BP}$, blood pressure; ECHO, echocardiogram; ENT, ear, nose and throat; HR, heart rate; MRA, magnetic resonance angiography. 
series. For most of our patients, especially those who were otherwise healthy, our original full therapeutic protocol is unnecessary as neither the pretreatment investigations nor the ongoing community based monitoring of BP and HR leads to deferment or alteration of the course of propranolol therapy. As a result, we have updated our treatment protocol (table 2).

Our updated treatment protocol is similar to the recommendations set by experienced group consensus, which was based on comprehensive literature review. ${ }^{20}$ The Consensus Report acknowledges that their recommendations are conservative in nature and anticipate revisions. There are minor differences between both protocols. The Consensus Report recommends outpatient initiation of propranolol therapy for patients older than 8 weeks corrected age, with adequate social support and without significant comorbidities. Our protocol recommends that patients are monitored for $2 \mathrm{~h}$ in the hospital and for $4 \mathrm{~h}$ or longer in patients weighting less than $3.5 \mathrm{~kg}$ or with comorbidities. Both protocols are in agreement with regard to pretreatment investigations, target dose, frequency and ongoing monitoring. As well, both protocols emphasise the importance of diagnosing PHACE syndrome, recognising the inherent risks involved in treating PHACE syndrome patients with propranolol and ensuring that that they are managed in close consultation with cardiology department. Our protocol extends even further by recommending when other investigations are indicated, as well as when to consult or refer to other specialties. Moreover, our protocol guides recommendations for the frequency of follow-up, the length of therapy and the appropriate manner to discontinue propranolol at the end of the treatment course.

\section{Updated treatment protocol}

Before initiation of propranolol for $\mathrm{IH}$, screening for risks associated with propranolol use should be performed. ${ }^{20}$ The prescribing physician should take complete and thorough history and perform physical examination with special emphasis on the signs and symptoms of cardiovascular and pulmonary disease. Pretreatment ECG and ECHO should be performed only in selected group of patients (table 2).

Contraindications for propranolol include cardiogenic shock, sinus bradycardia, hypotension, greater than first-degree heart block, heart failure, bronchial hypersensitivity and hypersensitivity to propranolol hydrochloride. ${ }^{20}$

Parents should be given information about the treatment and the possible adverse effects should be explained thoroughly. A leaflet should be provided including the direct contact number of the responsible team. Parents should be informed not to use lignocaine (usually used as a teething gel) and salbutamol. Propranolol has a potential to lower hepatic blood flow and may increase the toxicity of the lignocaine. ${ }^{21}$ Salbutamol is $\beta$-adrenergic receptor agonist; therefore, an alternative medication (eg, ipratropium bromide) should be used in case of bronchospasm. Parents should be instructed to ensure that their child is fed regularly to avoid prolonged fasts. Propranolol should be discontinued during intercurrent illness, especially in the setting of restricted oral intake. ${ }^{20}$

The starting dose of propranolol is $1 \mathrm{mg} / \mathrm{kg} /$ day in three divided doses. HR and BP are measured immediately before and then every $30 \mathrm{~min}$ for $2 \mathrm{~h}$ after administering the propranolol. Blood glucose is not measured routinely, unless the patient is having signs or symptoms of hypoglycaemia. For infants weighing less than $3.5 \mathrm{~kg}$ or with comorbidities, it may be appropriate to continue monitoring for $4 \mathrm{~h}$ or longer. This procedure is repeated after 1 week when the dose is increased to $2 \mathrm{mg} / \mathrm{kg} / \mathrm{day}$. In patients with suspected PHACE syndrome, it is prudent to start with the lower dose of propranolol- $0.5 \mathrm{mg} / \mathrm{kg} / \mathrm{day}$ in three divided doses and very cautiously increase the dose in small increments as propranolol may theoretically increase the risk of the stroke in these patients if arterial anomalies are present. After the magnetic resonance angiography (MRA) of the head and neck has been performed and arterial anomalies of head and neck have been excluded, the dose can be increased to $2 \mathrm{mg} / \mathrm{kg} /$ day.

In selected patients, additional investigations and referrals should be performed but the propranolol can be commenced while the results are pending. Thyroid function test should be done in patients with liver, parotid or very large haemangiomas to exclude consumptive hypothyroidism and in patients with suspected PHACE syndrome to exclude congenital hypothyroidism. LFT should be done in patients with liver haemangioma and FBC in patients with bleeding haemangiomas. Patients with more than 10 cutaneous lesions ${ }^{22}$ and patients with perianal/ perineal haemangioma crossing the midline and/or extending into a gluteal cleft should have an abdominal ultrasound. MRA of the head and neck should be done in patients with suspected PHACE syndrome. Patients with haemangioma in lumbosacral area or perianal haemangioma extending into gluteal cleft should have MRI of the spine. Patients with peri-ocular haemangioma should be referred to ophthalmology department and patients with suspected airway haemangioma should be referred to the ear, nose and throat team.

Ideally, the patient should be reviewed between 4 and 6 weeks after starting treatment, and then approximately 3-4 monthly. Monthly dose adjustment according to the weight of the child is needed unless the haemangioma is responding well to the treatment. Increments that are greater than $0.5 \mathrm{mg} / \mathrm{kg} / \mathrm{day}$ should include monitoring of the HR and BP for $2 \mathrm{~h}$. Occasionally, higher doses than $2 \mathrm{mg} / \mathrm{kg} /$ day of propranolol are required. If any adverse effects occur, the dose of propranolol should be temporarily withheld, lowered or the treatment should be stopped based on the clinical judgement of prescribing physician.

The time of cessation of growth of haemangiomas is very variable, and so the age when propranolol can be stopped, without further growth occurring, is also very variable. The treatment is usually stopped at 12-14 months of age, but substantially longer treatment may be required. When the decision has been made to take the child off propranolol, we recommend gradual dose reduction over 2-4 weeks.

Our experience has shown propranolol to be an effective and safe treatment for IH. On the basis of this experience, we have introduced a simplified therapeutic protocol.

Acknowledgements Department of Dermatology—Clinical Nurse Specialists: Jane Linward and Hilary Kennedy for their assistance.

Contributors $L S, A M, M G, S B S, J H H$ and MGl initiated the study design. $L S, A M$ and $M G$ collected the data. $L S, A M$ and $M G$ provided analysis and interpretation of the data. LS and AM drafted the manuscript. MG, SBS, JIH and MGI provided critical revision of the manuscript for important intellectual content.

Competing interests None.

Patient consent Obtained.

Provenance and peer review Not commissioned; externally peer reviewed.

\section{REFERENCES}

1 Haggstrom AN, Drolet BA, Baselga $E$, et al. Prospective study of infantile hemangiomas: demographic, prenatal, and perinatal characteristics. J Pediatr 2007;150:291-4.

2 Sundine MJ, Wirth GA. Hemangiomas: an overview. Clin Pediatr (Phila) 2007:46:206-21

3 Amir J, Metzker A, Krikler $R$, et al. Strawberry hemangioma in preterm infants. Pediatr Dermatol 1986;3:331-2. 


\section{Original article}

4 Frieden IJ, Haggstrom AN, Drolet BA, et al. Infantile hemangiomas: current knowledge future directions. Proceedings of a research workshop on infantile hemangiomas, April 7-9, 2005, Bethesda, Maryland, USA. Pediatr Dermatol 2005;22:383-406.

5 Haggstrom AN, Lammer EJ, Schneider RA, et al. Patterns of infantile hemangiomas: new clues to hemangioma pathogenesis and embryonic facial development. Pediatrics 2006;117:698-703.

6 Thomson HG, Lanigan M. The Cyrano nose: a clinical review of hemangiomas of the nasal tip. Plast Reconstr Surg 1979:63:155-60.

7 Chamlin SL, Haggstrom AN, Drolet BA, et al. Multicenter prospective study of ulcerated hemangiomas. J Pediatr 2007;151684-9, 89 e1.

8 Greene AK. Corticosteroid treatment for problematic infantile hemangioma: evidence does not support an increased risk for cerebral palsy. Pediatrics 2008;121:1251-2.

9 Fonseca Junior NL, Cha SB, Cartum J, et al. Therapeutical effectiveness of interferon alpha in a child with craniofacial giant hemangioma: case report. Arq Bras Oftalmol 2008;71:423-6.

10 Leaute-Labreze C, Dumas de la Roque E, Hubiche T, et al. Propranolol for severe hemangiomas of infancy. N Engl J Med 2008;358:2649-51.

11 Storch $\mathrm{CH}$, Hoeger PH. Propranolol for infantile haemangiomas: insights into the molecular mechanisms of action. Br J Dermatol 2010;163:269-74.

12 Balma-Mena A, Chakkittakandiyil A, Weinstein M, et al. Propranolol in the management of infantile hemangiomas: clinical response and predictors. J Cutan Med Surg 2012;16:169-73.
13 Fuchsmann C, Quintal MC, Giguere C, et al. Propranolol as first-line treatment of head and neck hemangiomas. Arch Otolaryngol Head Neck Surg 2011;137:471-8.

14 Manunza F, Syed S, Laguda B, et al. Propranolol for complicated infantile haemangiomas: a case series of 30 infants. Br J Dermatol 2010;162:466-8.

15 Visser CA, Garcia-Marcos L, Eggink J, et al. Prevalence and risk factors of wheeze in Dutch infants in their first year of life. Pediatr Pulmonol 2010;45:149-56.

16 Taussig LM, Wright AL, Holberg CJ, et al. Tucson Children's Respiratory Study: 1980 to present. J Allergy Clin Immunol 2003;111:661-75; quiz 76.

17 Maguiness SM, Frieden IJ. Management of difficult infantile haemangiomas. Arch Dis Child 2012;97:266-71.

18 Pavlakovic $\mathrm{H}$, Kietz $\mathrm{S}$, Lauerer $\mathrm{P}$, et al. Hyperkalemia complicating propranolol treatment of an infantile hemangioma. Pediatrics 2010;126:e1589-93.

19 Cavalli R, Buffon RB, de Souza M, et al. Tumor lysis syndrome after propranolo therapy in ulcerative infantile hemangioma: rare complication or incidental finding? Dermatology 2012;224:106-9.

20 Drolet BA, Frommelt PC, Chamlin SL, et al. Initiation and use of propranolol for infantile hemangioma: report of a consensus conference. Pediatrics 2013;131:128-40.

21 Bax ND, Tucker GT, Lennard MS, et al. The impairment of lignocaine clearance by propranolol--major contribution from enzyme inhibition. Br J Clin Pharmacol 1985;19:597-603.

22 Vredenborg $A D$, Janmohamed SR, de Laat PC, et al. Multiple cutaneous infantile haemangiomas and the risk of internal haemangioma. Br J Dermatol 2013;169:188-91. 